\title{
Young Age Is Associated with Increased Locoregional Recurrence in Node-Positive Breast Cancer with Luminal Subtypes
}

\author{
Sang-Won Kim, MD \\ Mison Chun, MD, PhD ${ }^{1}$ \\ Sehwan Han, MD, PhD² \\ Yong Sik Jung, MD2 \\ Jin Hyuk Choi, MD, PhD ${ }^{3}$ \\ Seok Yun Kang, MD, $\mathrm{PhD}^{3}$ \\ Hyunee Yim, MD, PhD ${ }^{4}$ \\ Seung Hee Kang, $M D^{5}$
}

Departments of ${ }^{1}$ Radiation Oncology,

${ }^{2}$ Surgery, ${ }^{3}$ Hematology-Oncology, and

${ }^{4}$ Pathology, Ajou University School of

Medicine, Suwon, ${ }^{5}$ Department of Radiation

Oncology, Ilsan Paik Hospital,

Inje University School of Medicine,

Goyang, Korea

Correspondence: Mison Chun, MD, PhD

Department of Radiation Oncology,

Ajou University School of Medicine,

206 Worldcup-ro, Yeongtong-gu,

Suwon 16499, Korea

Tel: 82-31-219-5884

Fax: 82-31-219-5894

E-mail: chunm@ajou.ac.kr

Received June 16, 2016

Accepted August 9, 2016

Published Online August 23, 2016

\begin{abstract}
Purpose
The effects of biological subtypes within breast cancer on prognosis are influenced by age at diagnosis. We investigated the association of young age with locoregional recurrence (LRR) between patients with luminal subtypes versus those with nonluminal subtypes.
\end{abstract}

\section{Materials and Methods}

Medical records of 524 breast cancer patients with positive lymph nodes between 1999 and 2010 were reviewed retrospectively. All patients received curative surgery and adjuvant chemotherapy based on contemporary guidelines. Radiation was delivered for patients who underwent breast conserving surgery or those who had four or more positive lymph nodes after mastectomy. Adjuvant hormone therapy was administered to 413 patients with positive hormone receptors according to their menstrual status.

\section{Results}

During median follow-up of 84 months, the 10-year locoregional recurrence-free survival rate (LRRFS) was $84.3 \%$ for all patients. Patients $<40$ years showed significantly worse 10 -year LRRFS than those $\geq 40$ years $(73.2 \%$ vs. $89.0 \%$, respectively; $p=0.01)$. The negative effect of young age on LRRFS was only observed in luminal subtypes $(69.7 \%$ for $<40$ years vs. $90.8 \%$ for $\geq 40$ years; $p<0.01$ ). Multivariate analysis using luminal subtypes $\geq 40$ years as a reference revealed luminal subtypes $<40$ years were significantly associated with increased risk of LRR (hazard ratio, 2.33; $p<0.01$ ).

\section{Conclusion}

Young breast cancer patients with positive lymph nodes had a higher risk of LRR than those aged $\geq 40$ years. This detrimental effect of young age on LRR was confined in luminal subtypes.

\section{Key words}

Breast neoplasms, Local neoplasm recurrence, Age factors

\section{Introduction}

Gene expression profiling identifies that breast cancer is a heterogeneous disease with at least four biological subtypes, representing distinct treatment responses and prognosis $[1,2]$. Because of its time-consuming process, cost-effectiveness and technical limitations of microarray on formalinfixed, paraffin-embedded tissue, gene expression profiling is difficult to apply routinely in clinical practice. Instead, a sim- plified method using combinations of estrogen receptor (ER), progesterone receptor (PR), and human epidermal growth factor receptor type 2 (HER2) measured by immunohistochemistry (IHC) as surrogate markers is widely accepted [3].

These surrogate markers provide prognostic information similar to that obtained by gene expression profiling $[4,5]$. However, previous studies have reported conflicting results when examining the influence of biological subtype as classified by IHC surrogate markers on locoregional recurrence (LRR), although there is a tendency toward an increased risk 
of LRR in HER2-enriched or triple negative subtypes [6-13].

Young age is well known to be related to poor prognosis and increased risk of LRR in breast cancer [14,15]. While investigating the effects of young age, several investigators found that young age influenced the prognosis of certain biological subtypes [16-20]. However, due to the retrospective nature of these studies, the results were inconsistent. While most studies focused on disease free or overall survival rate, the association of young age with LRR according to biological subtypes has not been sufficiently investigated, and only a few studies have been published $[17,18]$. We hypothesized that LRR in hormone receptor positive subtypes (luminal subtypes) would be significantly influenced by age after a review of related papers. The purpose of this study was to compare the effects of young age on LRR in luminal subtypes and nonluminal subtypes.

\section{Materials and Methods}

This retrospective study was approved by the Institutional Review Board of Ajou University School of Medicine, with a waiver of informed consent. Medical records of consecutive breast cancer patients with positive lymph nodes who received curative surgery and adjuvant chemotherapy at our institution between 1999 and 2010 were reviewed. Exclusion criteria for this study included (1) male breast cancer; (2) patients with distant metastasis at initial diagnosis; (3) patients who received preoperative treatment; (4) patients who did not receive adjuvant chemotherapy; (5) patients without information available for IHC staining; (6) synchronous bilateral invasive breast cancer; (7) a past history of previous malignancies, including metachronous contralateral breast cancer; and (8) patients without follow-up records. Using these criteria, 524 patients were identified.

All patients received curative surgery with either breast conserving surgery $(n=237)$ or mastectomy $(n=287)$. Axillary lymph node involvement was evaluated, and 22 patients received only sentinel lymph node biopsy because micrometastases were not found during intraoperative frozen biopsy.

All patients also received adjuvant chemotherapy. A taxane-based regimen was administered to 464 patients $(88.5 \%)$. Of the remaining 60 patients, 48 received anthracyclinebased chemotherapy, while the others received six cycles of cyclophosphamide, methotrexate, and fluorouracil. All patients with positive hormone receptor status received adjuvant endocrine therapy for 5 years, with the regimen determined by menopausal status.

Adjuvant radiotherapy was performed in 407 patients
(77.7\%) who received breast conserving surgery or had $\geq 4$ positive lymph nodes after mastectomy. Patients with T1-2 primary tumor and 1-3 positive lymph nodes after mastectomy did not receive postmastectomy radiotherapy. The ipsilateral breast or chest wall was irradiated using a tangential field with a median dose of 45 Gy in 1.8 to 2 Gy per fraction. An electron beam boost was delivered to tumor beds or surgical scars with 10 to $18 \mathrm{~Gy}$ in 2 to $2.5 \mathrm{~Gy}$ per fraction. The supraclavicular lymph node area was irradiated with a median dose of $45 \mathrm{~Gy}$ in patients with multiple positive lymph nodes. Internal mammary nodal irradiation was not performed routinely.

The results of IHC staining were obtained by pathologic reports. HER $2+$ was defined as an IHC score of $3+$ or $2+$ with gene amplification via fluorescent in situ hybridization (FISH). If the results of FISH were not available, an IHC score of $2+$ was regarded as negative $(n=22)$. Based on the results of IHC staining, patients were first dichotomized into luminal subtypes versus nonluminal subtypes, then classified into four subtypes: luminal A (ER or PR+, and HER2-), luminal B (ER or PR+, and HER2+), HER2-enriched (ER-, PR-, and HER2+), and triple negative (ER-, PR-, and HER2-).

\section{Endpoints and statistics}

The primary end-point of the present study was to investigate the effect of young age on LRR according to hormone receptor status. Comparison of locoregional outcomes between two age groups according to each biological subtype and identification of prognostic factors were the secondary end-points. The chi-square or Fisher exact tests were used to compare the clinical, disease and treatment-related variables between two age groups in this study, which were defined as $<40$ years and $\geq 40$ years. LRR was defined as the first tumor recurrence in the ipsilateral breast or chest wall, axillary lymph node, internal mammary lymph node and / or the infra-/supraclavicular lymph node area. The locoregional recurrence-free survival rate (LRRFS), defined as the period from the date of surgery to the date of diagnosis with LRR on imaging work-up, was calculated using the KaplanMeier method. The univariate log-rank test and multivariate Cox proportional hazard models were used to identify prognostic factors. Statistical analyses were performed using R software ver. 3.2.3 (https:// cran.r-project.org/) and IBM SPSS statistics for Windows ver. 20.0 (IBM Corp., Armonk, NY). 
Table 1. Patient characteristics

\begin{tabular}{|c|c|c|c|}
\hline Variable & Age $<40$ yr $(n=129)$ & Age $\geq 40$ yr $(n=395)$ & p-value \\
\hline \multicolumn{4}{|l|}{ Type of operation } \\
\hline Breast conserving & $53(41.1)$ & $184(46.6)$ & 0.31 \\
\hline Mastectomy & $76(58.9)$ & $211(53.4)$ & \\
\hline \multicolumn{4}{|l|}{ pT stage } \\
\hline $1-2$ & $113(87.6)$ & $360(91.1)$ & 0.30 \\
\hline $3-4$ & $16(12.4)$ & $35(8.9)$ & \\
\hline \multicolumn{4}{|l|}{ pN stage } \\
\hline 1 & $75(58.1)$ & $241(61.0)$ & 0.17 \\
\hline 2 & $23(17.8)$ & $87(22.0)$ & \\
\hline 3 & $31(24.0)$ & $67(17.0)$ & \\
\hline \multicolumn{4}{|l|}{ AJCC stage } \\
\hline II & $75(58.1)$ & $241(61.0)$ & 0.61 \\
\hline III & $54(41.9)$ & $154(39.0)$ & \\
\hline No. of dissected LN, mean & 20.8 & 20.1 & 0.36 \\
\hline \multicolumn{4}{|l|}{ Lymph node ratio } \\
\hline$<0.2$ & $72(55.8)$ & $236(59.7)$ & 0.47 \\
\hline$\geq 0.2$ & $57(44.2)$ & $159(40.3)$ & \\
\hline \multicolumn{4}{|l|}{ Resection margin (mm) } \\
\hline$\geq 2$ & $105(81.4)$ & $326(82.5)$ & 0.77 \\
\hline$<2$ & $24(18.6)$ & $69(17.5)$ & \\
\hline \multicolumn{4}{|l|}{ Histologic grade } \\
\hline Low & $9(7.0)$ & $34(8.6)$ & 0.62 \\
\hline Intermediate & $44(34.1)$ & $155(39.2)$ & \\
\hline High & $70(54.3)$ & $189(47.8)$ & \\
\hline Unknown & $6(4.7)$ & $17(4.3)$ & \\
\hline \multicolumn{4}{|l|}{ Biological subtype } \\
\hline Luminal A (ER or PR+, HER2-) & $65(50.4)$ & $239(60.5)$ & $<0.01$ \\
\hline Luminal B (ER or PR+, HER2+) & $43(33.3)$ & $66(16.7)$ & \\
\hline HER2 (ER-, PR-, HER2+) & $12(9.3)$ & $43(10.9)$ & \\
\hline TN (ER-, PR-, HER2-) & $9(7.0)$ & 47 (11.9) & \\
\hline \multicolumn{4}{|l|}{ Adjuvant radiotherapy } \\
\hline No & $27(20.9)$ & $90(22.8)$ & 0.23 \\
\hline Breast/Chest wall only & $21(16.3)$ & $93(23.5)$ & \\
\hline Breast/Chest wall+SCL & $50(38.8)$ & $138(34.9)$ & \\
\hline Breast/Chest wall+SCL+IMN & $31(24.0)$ & $74(18.7)$ & \\
\hline \multicolumn{4}{|l|}{ Adjuvant trastuzumab ${ }^{a}$} \\
\hline No & $50(90.9)$ & $78(71.6)$ & $<0.01$ \\
\hline Yes & $5(9.1)$ & $31(28.4)$ & \\
\hline
\end{tabular}

Values are presented as number (\%). AJCC, American Joint Committee on Cancer; LN, lymph node; ER, estrogen receptor; PR, progesterone receptor; HER2, human epidermal growth factor receptor type 2; TN, triple negative; SCL, supraclavicular

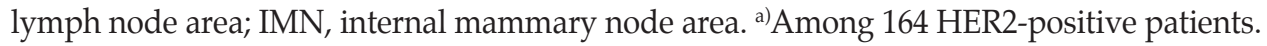


Table 2. Incidence of locoregional recurrence

\begin{tabular}{|ccc|}
\hline Variable & Age $<40$ yr & Age $\geq 40$ yr \\
\hline Total & $23(17.8)$ & $35(8.9)$ \\
\hline Luminal A & $12(18.5)$ & $13(5.4)$ \\
\hline Luminal B & $10(23.3)$ & $10(15.2)$ \\
\hline HER2-enriched & $1(8.3)$ & $7(16.3)$ \\
\hline Triple negative & 0 & $5(10.6)$ \\
\hline Local & & \\
\hline Luminal A & $7(10.8)$ & $4(1.7)$ \\
\hline Luminal B & $3(7.0)$ & $4(6.1)$ \\
\hline HER2-enriched & $1(8.3)$ & $3(7.0)$ \\
\hline Triple negative & 0 & $3(6.4)$ \\
\hline Regional & & \\
\hline Luminal A & $6(9.2)$ & $9(3.8)$ \\
\hline Luminal B & $7(16.3)$ & $7(10.6)$ \\
\hline HER2-enriched & 0 & $5(11.6)$ \\
\hline Triple negative & 0 & $3(6.4)$ \\
\hline
\end{tabular}

Values are presented as number (\%). HER2, human epidermal growth factor receptor type 2 .

\section{Results}

\section{Patient characteristics}

The information and characteristics for the 524 patients reviewed for this study are summarized in Table 1. The median age for the entire patient population was 45 years (range, 22 to 80 years), with 129 patients $(24.6 \%$ ) less than 40 years. Luminal A subtype was the most common subtype $(\mathrm{n}=304,58.0 \%)$, followed by luminal B $(\mathrm{n}=109,20.8 \%)$, triple negative $(n=56,10.7 \%)$, and HER2-enriched subtype $(n=55$, 10.5\%). No pathological factors except biological subtypes were significantly different between age groups. More patients in the older age group received adjuvant trastuzumab. This difference in proportion of patients receiving adjuvant trastuzumab was significant only in the luminal B subtype (three patients [7.0\%] in $<40$ years vs. 19 patients [28.8\%] in $\geq 40$ years, $\mathrm{p}<0.01)$.

\section{Locoregional recurrence}

During the median follow up period of 84 months, LRR developed in 58 patients (11.1\%) (Table 2). Of these, LRR occurred in 23 patients $(17.8 \%)$ for $<40$ years and 35 patients $(8.9 \%)$ for $\geq 40$ years. According to hormone receptor status, LRR occurred in 45 patients (10.9\%) for luminal subtypes (8.2\% for luminal A and $18.3 \%$ for luminal B) and 13 patients $(11.7 \%)$ for nonluminal subtypes (14.5\% for HER2-enriched and $8.9 \%$ for triple negative subtype).

The 10-year LRRFS for all patients was 84.3\% (Fig. 1A). Age was significantly associated with the 10-year LRRFS (73.2\% for $<40$ years vs. $89.0 \%$ for $\geq 40$ years, $\mathrm{p}<0.01$ ) (Fig. 1B). In contrast, the 10-year LRRFS was not significantly different between luminal and nonluminal subtypes (85.0\% vs. $84.3 \%$, respectively; $p=0.50$ ) (Fig. 1C). Subgroup analysis revealed that the 10-year LRRFS for each biological subtype was $86.6 \%$ for luminal A, $77.4 \%$ for luminal B, $81.5 \%$ for HER2 and $88.4 \%$ for the triple negative subtype $(p=0.05)$ (Fig. 1D).

The effect of young age on LRRFS was compared between luminal and nonluminal subtypes. In luminal subtypes, the 10 -year LRRFS was significantly lower for patients aged $<40$ years when compared to those aged $\geq 40$ years in the luminal subtypes $(69.7 \%$ vs. $90.8 \%$, respectively; $\mathrm{p}<0.01)$. In contrast, the 10-year LRRFS was $95.0 \%$ for patients aged $<40$ years and $82.7 \%$ for those $\geq 40$ years in nonluminal subtypes $(\mathrm{p}=0.29)$ (Fig. 2A and B).

Subgroup analysis to determine the effect of young age on LRRFS according to each biological subtype revealed that the 10-year LRRFS was $67.5 \%$ for $<40$ years versus $92.9 \%$ for $\geq 40$ years in luminal A ( $p<0.01), 70.8 \%$ for $<40$ years versus $82.9 \%$ for $\geq 40$ years in luminal B ( $p=0.55), 91.7 \%$ for $<40$ years versus $77.9 \%$ for $\geq 40$ years in HER2-enriched ( $\mathrm{p}=0.42$ ), and $100 \%$ for $<40$ years versus $86.7 \%$ for $\geq 40$ years in the triple negative subtype ( $\mathrm{p}=0.36$ ) (Fig. 2C-F).

In addition to young age, histologic grade and resection margin $<2$ mm were important prognostic factors for LRRFS upon univariate analysis (all $\mathrm{p}<0.01)$. However, $\mathrm{T}$ stage (1-2 vs. $3-4)$, N stage (1 vs. 2 vs. 3 ), type of breast surgery (breast conserving surgery vs. mastectomy), lymph node ratio ( $<0.2$ vs. $\geq 0.2$ ), radiation field and use of adjuvant trastuzumab were not significantly associated with LRRFS (Table 3). Young age was associated with lower LRRFS upon multivariate analysis with adjustment of pathological $\mathrm{T}$ stage, nodal status, histologic grade, resection margin status and biological subtypes (hazard ratio [HR], 1.92; 95\% confidence interval [CI], 1.11 to $3.35 ; \mathrm{p}=0.02$ ).

After multivariate analysis, which used luminal subtypes for $\geq 40$ years as the reference, luminal subtypes for $<40$ years were significantly associated with a lower LRRFS (HR, 2.33; 95\% CI, 1.28 to $4.22 ; \mathrm{p}<0.01$ ) (Table 4). Additional multivariate analysis, which used luminal $\mathrm{A} \geq 40$ years as the reference, revealed that both luminal A (HR, 2.87; 95\% CI, 1.29 to 6.37; $\mathrm{p}=0.01$ ) and luminal $\mathrm{B}$ for $<40$ years (HR, 3.58; $95 \%$ 1.50 to $8.54 ; \mathrm{p}<0.01$ ) were independent factors for a lower LRRFS. 
A
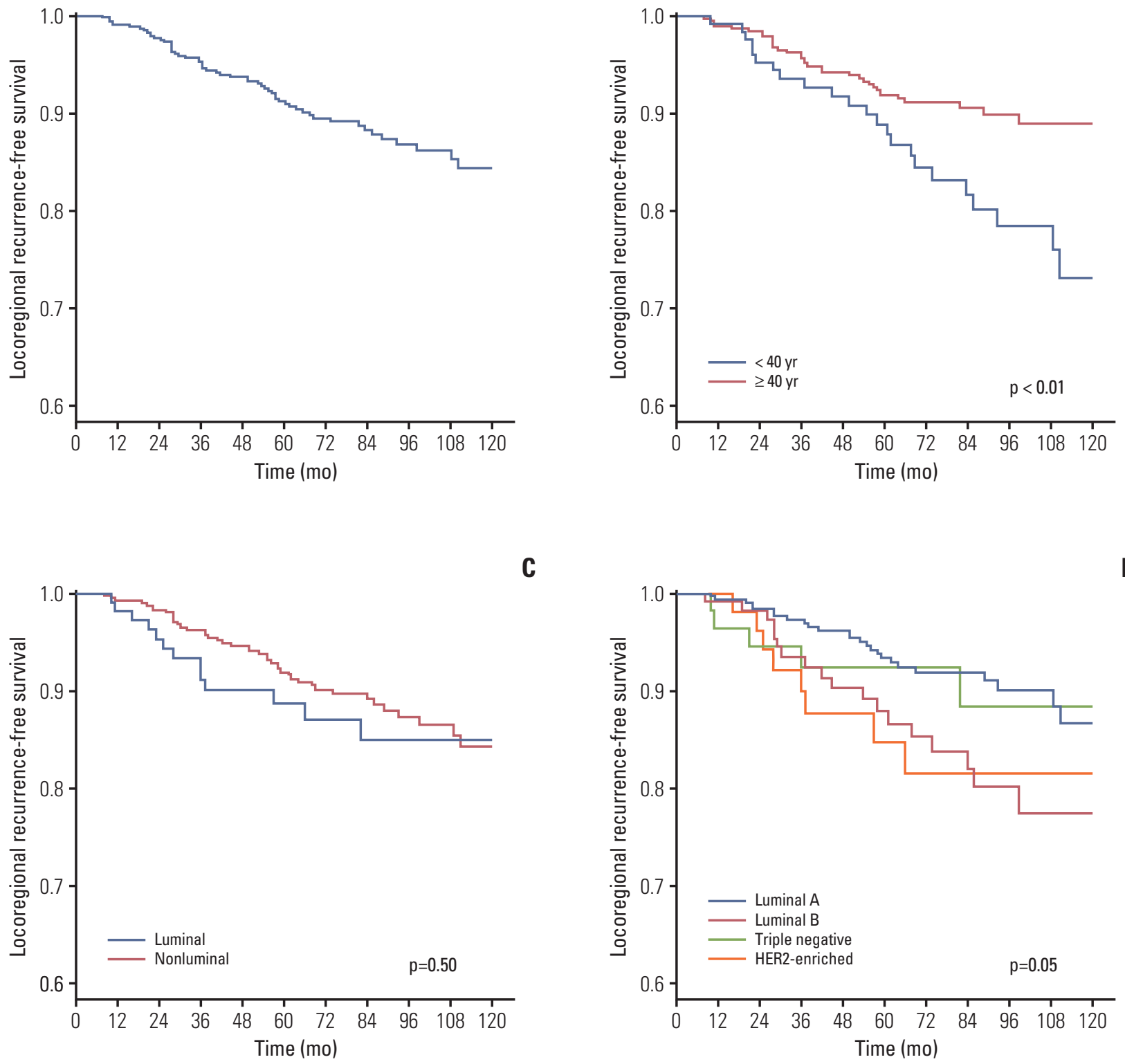

Fig. 1. The 10-year locoregional recurrence-free survival rate for all patients (A), between those aged $<40$ years and $\geq 40$ years (B), between luminal and nonluminal subtypes (C), and between biological subtypes (D).

\section{Discussion}

The results of our study showed that young age was significantly associated with lower LRRFS. Young age patients in our study did not present with any of the high risk pathologic features such as advanced stage or high grade, which was contrary to earlier studies [21]. Nonetheless, the LRRFS of young breast cancer patients was significantly lower than that of older patients. After adjusting all possible prognostic factors on LRRFS, including pathological $\mathrm{T}$ stage, nodal status, histologic grade, resection margin status and biological subtypes, young age remained an independent factor on LRRFS.

More importantly, the negative effect of young age on LRRFS was observed only in the luminal subtypes. In particular, the LRRFS of luminal A subtype was seriously influenced by young age. In contrast, young age did not have a 


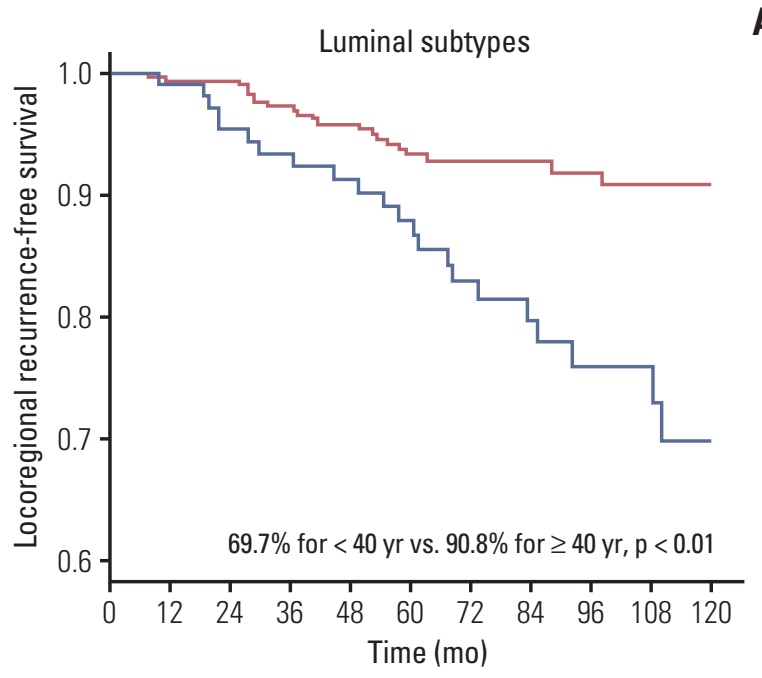

A
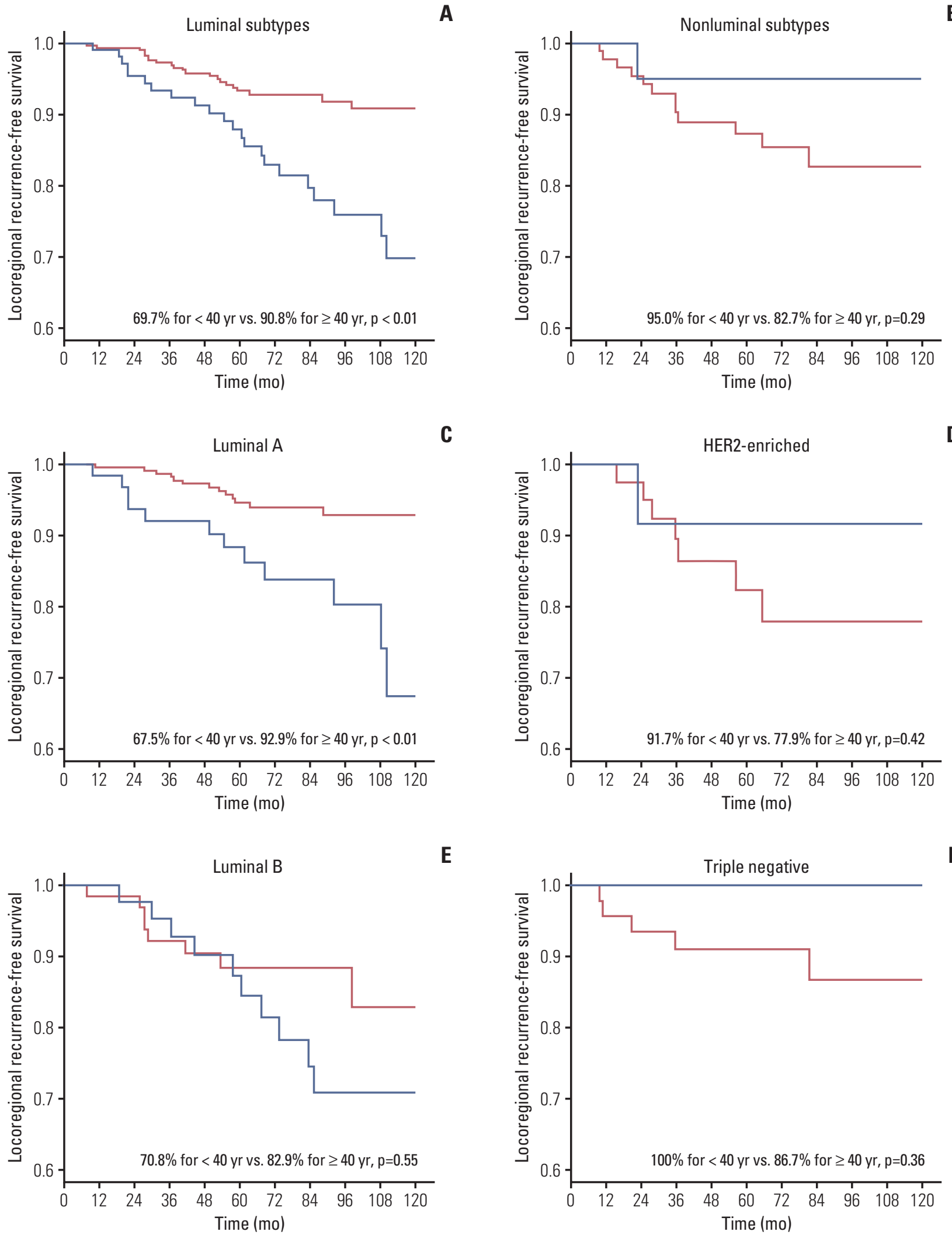

$\mathbf{E}$

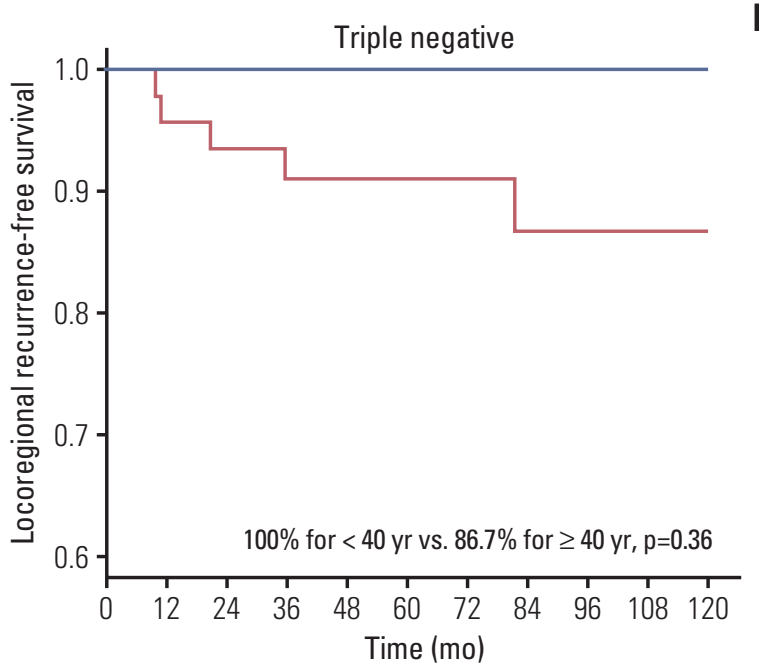

Fig. 2. The 10-year locoregional recurrence-free survival rate between patients aged $<40$ years (blue line) and $\geq 40$ years (red line) for luminal subtypes (A), nonluminal subtypes (B), luminal A (C), human epidermal growth factor receptor type 2 (HER2)-enriched (D), luminal B (E), and triple negative subtypes (F). 
Table 3. Univariate analysis for LRRFS

\begin{tabular}{|c|c|c|}
\hline Variable & 10-Yr LRRFS (\%) & p-value \\
\hline \multicolumn{3}{|l|}{ Age (yr) } \\
\hline$<40$ & 73.2 & 0.01 \\
\hline$\geq 40$ & 89 & \\
\hline \multicolumn{3}{|l|}{ Type of breast surgery } \\
\hline Breast conserving surgery & 86.5 & 0.17 \\
\hline Total mastectomy & 82.5 & \\
\hline \multicolumn{3}{|l|}{ T stage } \\
\hline pT1-2 & 84.0 & 0.91 \\
\hline pT3-4 & 89.0 & \\
\hline \multicolumn{3}{|l|}{ N stage } \\
\hline 1 & 85.2 & 0.26 \\
\hline 2 & 80.4 & \\
\hline 3 & 85.4 & \\
\hline \multicolumn{3}{|l|}{ Lymph node ratio } \\
\hline$<0.2$ & 84.9 & 0.09 \\
\hline$\geq 0.2$ & 83.6 & \\
\hline \multicolumn{3}{|l|}{ Histologic grade } \\
\hline 1 (low) & 100 & $<0.01$ \\
\hline 2 (intermediate) & 87.8 & \\
\hline 3 (high) & 77.5 & \\
\hline \multicolumn{3}{|l|}{ Resection margin (mm) } \\
\hline$<2$ & 87.4 & $<0.01$ \\
\hline$\geq 2$ & 69.2 & \\
\hline \multicolumn{3}{|l|}{ Biological subtype } \\
\hline Luminal A & 86.6 & 0.05 \\
\hline Luminal B & 77.4 & \\
\hline HER2-enriched & 81.5 & \\
\hline Triple negative & 88.4 & \\
\hline \multicolumn{3}{|l|}{ Radiation field } \\
\hline No & 79.5 & 0.42 \\
\hline Breast/Chest wall only & 85.7 & \\
\hline Breast/Chest wall+SCL & 84 & \\
\hline Breast/Chest wall+SCL+IMN & 89.1 & \\
\hline \multicolumn{3}{|l|}{ Adjuvant trastuzumab } \\
\hline No & 76 & 0.24 \\
\hline Yes & 91.2 & \\
\hline
\end{tabular}

LRRFS, locoregional recurrence-free survival; HER2, human epidermal growth factor receptor type 2; SCL, supraclavicular lymph node area; IMN, internal mammary node area.

significant impact on LRRFS in nonluminal subtypes.

The detrimental effect of young age on the prognosis of luminal subtypes in our study was concordant with the results of previous studies $[16,22]$. For example, Colleoni et al. [22] reported that the adverse effects of young age on disease-free and overall survival rates was observed mainly in hormone receptor-positive breast cancer. Ahn et al. [16] reported similar results upon analysis of data from a nationwide breast cancer registry. Their study showed that young age significantly reduced survival rates only in hormone receptor-positive breast cancer, but not in hormone receptornegative breast cancer. The most notable finding from the study conducted by Ahn et al. [16] was the lack of survival benefit from adjuvant hormone therapy in young patients with positive hormone receptor breast cancer. The survival outcomes between patients who received adjuvant tamoxifen and those who did not were not significantly different in young age breast cancer patients with luminal subtypes. This lack of benefit from adjuvant tamoxifen might be an important cause for unfavorable survival rates in young age breast cancer patients compared with older patients in luminal subtypes.

Because all patients with luminal subtypes in our study received adjuvant hormone therapy, we could not directly assess the LRRFS benefit of selective ER modulators. However, it could be implied that, because the results of our study were similar to those of Ahn et al. [16], less effective adjuvant hormone therapy might also contribute to lower LRRFS in young patients with luminal subtypes compared with older patients. The duration of tamoxifen use for 5 years in our study might explain the ineffectiveness of adjuvant hormone therapy. Indeed, a recent large prospective randomized trial showed improvement in clinical outcomes when continuing tamoxifen to 10 years compared with stopping at 5 years [23]. However, the rate of LRRFS in young breast cancer patients with luminal subtypes decreased constantly over all periods, regardless of duration of adjuvant hormone therapy. Therefore, duration of adjuvant hormone therapy does not appear to matter, and the reason for lack of benefit from adjuvant hormone therapy in young breast cancer patients is still not fully understood.

Several researchers have reported an association between young age and risk of LRR according to each biological subtype $[17,18]$. Cancello et al. [17] reported that young age was an independent factor for increased breast cancer related events, including LRR in luminal B subtype (ER and / or PR+ and [Ki-67 $\geq 14 \%$ or HER2+]). In addition, Kim et al. [18] reported that young age significantly increased ipsilateral breast tumor recurrence, mainly in the HER2-enriched subtype. Although luminal A subtype in young patients also increased ipsilateral breast tumor recurrence when compared with old patients, the difference showed borderline significance upon multivariate analysis $(\mathrm{HR}, 2.97 ; \mathrm{p}=0.053)$. These two studies commonly demonstrated that young age increased risk of LRR in luminal subtypes, regardless of HER2 status. Similarly, the LRRFS of luminal subtypes in our study, particularly luminal A subtype, was significantly influenced by young age.

In most previous studies, luminal A subtype showed the 
Table 4. Multivariate Cox proportional hazards regression models for LRRFS

\begin{tabular}{|c|c|c|c|}
\hline Variable & HR & $95 \% \mathrm{CI}$ & p-value \\
\hline \multicolumn{4}{|c|}{ Luminal vs. nonluminal } \\
\hline \multicolumn{4}{|c|}{ Age $\geq 40 \mathrm{yr}$} \\
\hline Luminal & 1.00 & & \\
\hline Nonluminal & 1.40 & $0.65-3.01$ & 0.39 \\
\hline \multicolumn{4}{|l|}{ Age $<40$ yr } \\
\hline Luminal & 2.33 & $1.28-4.22$ & $<0.01$ \\
\hline Nonluminal & 0.66 & $0.09-4.95$ & 0.68 \\
\hline \multicolumn{4}{|c|}{ Four biological subtypes } \\
\hline \multicolumn{4}{|c|}{ Age $\geq 40$ yr } \\
\hline Luminal A & 1.00 & & \\
\hline Luminal B & 2.33 & $1.00-5.42$ & 0.05 \\
\hline HER2-enriched & 2.46 & $0.91-6.66$ & 0.08 \\
\hline Triple negative & 1.37 & $0.44-4.29$ & 0.59 \\
\hline \multicolumn{4}{|l|}{ Age $<40$ yr } \\
\hline Luminal A & 2.87 & $1.29-6.37$ & 0.01 \\
\hline Luminal B & 3.58 & $1.50-8.54$ & $<0.01$ \\
\hline HER2-enriched & 1.57 & $0.20-12.27$ & 0.67 \\
\hline Triple negative & 0 & 0 & 0.96 \\
\hline
\end{tabular}

LRRFS, locoregional recurrence free survival rate; HR, hazard ratio; CI, confidence interval; HER2, human epidermal growth factor receptor type 2 .

lowest incidence rate of LRR among biological subtypes [8-12]. However, in our study, the 10-year LRRFS in luminal A subtype was not more favorable than the other subtypes in all patients, particularly young patients. One possible explanation for this observation was the possibility of a high Ki-67 index. This marker is now incorporated into the classification of biological subtypes that differentiate luminal A (ER and/or PR+, HER2-, and Ki-67 < 14\%) from luminal B (ER and/or PR+, HER2-, and Ki-67 $\geq 14 \%$ ) [24]. The existing luminal B, which was defined as ER and/or PR+ and HER2+, is then renamed as luminal HER2. Indeed, the definition of luminal B by HER2 is not appropriate, because only 30\%-50\% of luminal B subtype identified by gene expression profiling overexpresses HER2. Therefore, a considerable proportion of luminal A subtype in our study might actually be classified into the current luminal B subtype (ER and/or PR+, HER2-, and high Ki-67). The higher level of the Ki-67 index in young age breast cancer patients with positive hormone receptors supported this possibility [25]. However, measurement of the Ki-67 index was not available for the period of time in which this study collected patient data. Although histologic grade can be used as a good alternative for Ki-67 [26], we did not classify biological subtypes with this marker because this information was not provided for approximately $5 \%$ of patients.

Equivocal definition of HER2 overexpression could also be an explanation for the relatively lower LRRFS for luminal A subtype. In our study, 22 patients who showed HER2 scoring of $2+$ without FISH were regarded as negative and classified as luminal A or triple negative subtype. Approximately $20 \%$ $30 \%$ of HER 2 scoring of $2+$ were known to present overexpression via FISH [5]. Accordingly, some luminal A subtype patients might have been classified into luminal B subtype if FISH was conducted. However, only one patient with HER2 scoring of $2+$ experienced LRR. Therefore, it was unlikely that re-classification of biological subtypes by accurate assessment of HER2 status with FISH would change LRRFS between each subtype.

Another possible reason for the relatively lower LRRFS for luminal A subtype was that the median age in our study was relatively young for the entire population when compared with previous studies. In particular, the luminal subtypes in the majority of previous studies showed a tendency towards older age when compared with the triple negative subtype, which is known for its high frequency in young age. However, in the present study, the median age was 45 years for luminal subtypes (45 years for luminal A and 42 years for luminal B) and 47 years for nonluminal subtypes. As young age was an independent factor for lower LRRFS in luminal subtypes, the age distribution of patients in our study might contribute to the contradictory results observed when compared to previous studies $[8,9,13]$. 
One interesting result of our study was the comparable outcomes for triple negative subtype breast cancer in young patients. Triple negative subtype is known to be associated with an increased risk of LRR when compared with luminal subtypes [8-13]. However, triple negative subtype in young breast cancer patients did not appear to have a worse prognosis than luminal subtypes $[20,27]$. Moreover, the negative effects of young age on prognosis for triple negative subtype breast cancer have been controversial $[18,19,27,28]$. Our study demonstrated that triple negative subtype was not associated with lower LRRFS in young patients, and that the LRRFS for triple negative subtype was not influenced by young age. However, these results should be confirmed by further studies with well designed and large scale.

Our study had several limitations. First, owing to the retrospective nature of the study, selection bias was inevitable. The small number of cases, especially of young age with nonluminal subtypes, led to insufficient statistical power and hindered interpretation of our results. Additionally, we could only investigate node-positive breast cancer patients because poor survival outcomes associated with young age was more remarkable in node-positive breast cancer [29]. Classification of biological subtypes by only three IHC surrogate markers was also an important limitation of our study because these markers are only an approximation of the underlying genotype-based subtypes. In addition to these markers, Ki-67 index, cytokeratin 5/6, and epidermal growth factor receptor levels are currently used to identify breast cancer subtypes, and these six biomarkers more accurately reflect breast cancer subtypes based on gene expression profiling [30]. Therefore, our results might not be reproduced using genotype-based subtypes.

The presence of a small proportion of patients who did not receive taxane-based adjuvant chemotherapy or who received adjuvant trastuzumab also represented a potential limitation. Furthermore, patients receiving adjuvant trastuzumab were more common in the older age group. This could influence the minor difference of LRRFS between age groups in the luminal B subtype. However, the significant difference in LRRFS in all patients with luminal subtypes was predominantly determined by luminal A subtype in the present study. When we re-analyzed the outcomes of patients with luminal subtypes by excluding those treated with adjuvant trastuzumab, the results did not change (data not shown). It should also be noted that, in the HER2-enriched subgroup, the LRRFS of the young age group tended to be more favorable than that of the older age group $(91.7 \%$ vs. $77.9 \%$, respectively), even though patients treated with adjuvant trastuzumab were more common in the older age group. Furthermore, univariate analysis showed that adjuvant taxane and trastuzumab were not associated with lower LRRFS, Therefore, it could be assumed that the benefit of adjuvant trastuzumab did not overcome the effects of age, particularly in luminal subtypes.

\section{Conclusion}

In summary, our study demonstrated that young age represented an important risk factor for LRRFS in node-positive breast cancer, and that its detrimental effect was confined to the luminal subtypes. As all patients received uniform multimodality treatments based on contemporary guidelines, we suggest that novel tailored adjuvant local and systemic treatments are required for young breast cancer patients with luminal subtypes. In addition, further investigations to identify unique genotypic characteristics in young breast cancer patients are warranted.

\section{Conflicts of Interest}

Conflict of interest relevant to this article was not reported.

\section{References}

1. Perou CM, Sorlie T, Eisen MB, van de Rijn M, Jeffrey SS, Rees CA, et al. Molecular portraits of human breast tumours. Nature. 2000;406:747-52.

2. Sorlie T, Perou CM, Tibshirani R, Aas T, Geisler S, Johnsen H, et al. Gene expression patterns of breast carcinomas distinguish tumor subclasses with clinical implications. Proc Natl Acad Sci U S A. 2001;98:10869-74.
3. Brenton JD, Carey LA, Ahmed AA, Caldas C. Molecular classification and molecular forecasting of breast cancer: ready for clinical application? J Clin Oncol. 2005;23:7350-60.

4. Nielsen TO, Hsu FD, Jensen K, Cheang M, Karaca G, Hu Z, et al. Immunohistochemical and clinical characterization of the basal-like subtype of invasive breast carcinoma. Clin Cancer Res. 2004;10:5367-74. 
5. Carey LA, Perou CM, Livasy CA, Dressler LG, Cowan D, Conway $\mathrm{K}$, et al. Race, breast cancer subtypes, and survival in the Carolina Breast Cancer Study. JAMA. 2006;295:2492-502.

6. Haffty BG, Yang Q, Reiss M, Kearney T, Higgins SA, Weidhaas $\mathrm{J}$, et al. Locoregional relapse and distant metastasis in conservatively managed triple negative early-stage breast cancer. J Clin Oncol. 2006;24:5652-7.

7. Kyndi M, Sorensen FB, Knudsen H, Overgaard M, Nielsen $\mathrm{HM}$, Overgaard J. Estrogen receptor, progesterone receptor, HER-2, and response to postmastectomy radiotherapy in highrisk breast cancer: the Danish Breast Cancer Cooperative Group. J Clin Oncol. 2008;26:1419-26.

8. Nguyen PL, Taghian AG, Katz MS, Niemierko A, Abi Raad RF, Boon WL, et al. Breast cancer subtype approximated by estrogen receptor, progesterone receptor, and HER-2 is associated with local and distant recurrence after breast-conserving therapy. J Clin Oncol. 2008;26:2373-8.

9. Millar EK, Graham PH, O'Toole SA, McNeil CM, Browne L, Morey AL, et al. Prediction of local recurrence, distant metastases, and death after breast-conserving therapy in early-stage invasive breast cancer using a five-biomarker panel. J Clin Oncol. 2009;27:4701-8.

10. Voduc KD, Cheang MC, Tyldesley S, Gelmon K, Nielsen TO, Kennecke H. Breast cancer subtypes and the risk of local and regional relapse. J Clin Oncol. 2010;28:1684-91.

11. Gabos Z, Thoms J, Ghosh S, Hanson J, Deschenes J, Sabri S, et al. The association between biological subtype and locoregional recurrence in newly diagnosed breast cancer. Breast Cancer Res Treat. 2010;124:187-94.

12. Arvold ND, Taghian AG, Niemierko A, Abi Raad RF, Sreedhara M, Nguyen PL, et al. Age, breast cancer subtype approximation, and local recurrence after breast-conserving therapy. J Clin Oncol. 2011;29:3885-91.

13. Metzger-Filho O, Sun Z, Viale G, Price KN, Crivellari D, Snyder RD, et al. Patterns of recurrence and outcome according to breast cancer subtypes in lymph node-negative disease: results from international breast cancer study group trials VIII and IX. J Clin Oncol. 2013;31:3083-90.

14. Adami HO, Malker B, Holmberg L, Persson I, Stone B. The relation between survival and age at diagnosis in breast cancer. N Engl J Med. 1986;315:559-63.

15. Anders CK, Hsu DS, Broadwater G, Acharya CR, Foekens JA, Zhang $\mathrm{Y}$, et al. Young age at diagnosis correlates with worse prognosis and defines a subset of breast cancers with shared patterns of gene expression. J Clin Oncol. 2008;26:3324-30.

16. Ahn SH, Son BH, Kim SW, Kim SI, Jeong J, Ko SS, et al. Poor outcome of hormone receptor-positive breast cancer at very young age is due to tamoxifen resistance: nationwide survival data in Korea: a report from the Korean Breast Cancer Society. J Clin Oncol. 2007;25:2360-8.

17. Cancello G, Maisonneuve P, Rotmensz N, Viale G, Mastropasqua MG, Pruneri G, et al. Prognosis and adjuvant treatment effects in selected breast cancer subtypes of very young women $(<35$ years) with operable breast cancer. Ann Oncol.
2010;21:1974-81.

18. Kim HJ, Han W, Yi OV, Shin HC, Ahn SK, Koh BS, et al. Young age is associated with ipsilateral breast tumor recurrence after breast conserving surgery and radiation therapy in patients with HER2-positive/ER-negative subtype. Breast Cancer Res Treat. 2011;130:499-505.

19. Kim EK, Noh WC, Han W, Noh DY. Prognostic significance of young age ( $<35$ years) by subtype based on ER, PR, and HER2 status in breast cancer: a nationwide registry-based study. World J Surg. 2011;35:1244-53.

20. Park YH, Lee SJ, Jung HA, Kim SM, Kim MJ, Kil WH, et al. Prevalence and clinical outcomes of young breast cancer (YBC) patients according to intrinsic breast cancer subtypes: Single institutional experience in Korea. Breast. 2015;24:213-7.

21. Albain KS, Allred DC, Clark GM. Breast cancer outcome and predictors of outcome: are there age differentials? J Natl Cancer Inst Monogr. 1994;(16):35-42.

22. Colleoni M, Rotmensz N, Peruzzotti G, Maisonneuve P, Orlando L, Ghisini R, et al. Role of endocrine responsiveness and adjuvant therapy in very young women (below 35 years) with operable breast cancer and node negative disease. Ann Oncol. 2006;17:1497-503.

23. Davies C, Pan H, Godwin J, Gray R, Arriagada R, Raina V, et al. Long-term effects of continuing adjuvant tamoxifen to 10 years versus stopping at 5 years after diagnosis of oestrogen receptor-positive breast cancer: ATLAS, a randomised trial. Lancet. 2013;381:805-16.

24. Cheang MC, Chia SK, Voduc D, Gao D, Leung S, Snider J, et al. Ki67 index, HER2 status, and prognosis of patients with luminal B breast cancer. J Natl Cancer Inst. 2009;101:736-50.

25. Kim J, Han W, Jung SY, Park YH, Moon HG, Ahn SK, et al. The value of Ki67 in very young women with hormone receptor-positive breast cancer: retrospective analysis of 9,321 Korean women. Ann Surg Oncol. 2015;22:3481-8.

26. Goldhirsch A, Wood WC, Coates AS, Gelber RD, Thurlimann B, Senn HJ. Strategies for subtypes: dealing with the diversity of breast cancer: highlights of the St. Gallen International Expert Consensus on the Primary Therapy of Early Breast Cancer 2011. Ann Oncol. 2011;22:1736-47.

27. Sheridan W, Scott T, Caroline S, Yvonne Z, Vanessa B, David $\mathrm{V}$, et al. Breast cancer in young women: have the prognostic implications of breast cancer subtypes changed over time? Breast Cancer Res Treat. 2014;147:617-29.

28. Liedtke C, Hess KR, Karn T, Rody A, Kiesel L, Hortobagyi GN, et al. The prognostic impact of age in patients with triple-negative breast cancer. Breast Cancer Res Treat. 2013;138:591-9.

29. Han W, Kim SW, Park IA, Kang D, Youn YK, Oh SK, et al. Young age: an independent risk factor for disease-free survival in women with operable breast cancer. BMC Cancer. 2004;4:82.

30. Cheang MC, Voduc D, Bajdik C, Leung S, McKinney S, Chia $\mathrm{SK}$, et al. Basal-like breast cancer defined by five biomarkers has superior prognostic value than triple-negative phenotype. Clin Cancer Res. 2008;14:1368-76. 